\title{
UMA ABORDAGEM CONTINGENCIAL DE GERENCIAMENTO DE PROJETOS NO DESENVOLVIMENTO DE UMA FERRAMENTA DE APOIO À GESTÃO DA PÓS-GRADUAÇÃO STRICTO SENSU
}

Doi 10.21902/emprad.v1i1.249

Recebido: $23 / 08 / 2016$

Aprovado: 24/10/2016

\author{
Ricardo Antônio Câmara da Silva ${ }^{1}$ \\ Emerson Maccari² \\ Renato Ribeiro Nogueira Ferraz ${ }^{3}$
}

\section{Resumo}

Este trabalho constitui-se em uma pesquisa aplicada, qualitativa e exploratória, realizada pelo método da pesquisa-ação. Tem como objetivo investigar a aplicação de uma abordagem contingencial no gerenciamento de um projeto destinado a desenvolver uma ferramenta com aplicação prática, no apoio à gestão da Pós-Graduação Stricto sensu. Na fase inicial do projeto foi escolhida a abordagem Adaptativa, por meio da aplicação do método do Ciclo de Vida de Gerenciamento de Projetos. O trabalho do projeto compreende a integração de dados armazenado em bancos de dados dos órgãos governamentais CAPES e CNPq, assim como a construção de um protótipo que disponibilize consultas aos dados integrados e permita a geração automatizada de listas de entrada para o programa Scriptsucupira. A implementação da ferramenta proposta facilitará a recuperação mais eficiente da produção científica dos docente brasileiros, contribuindo para a geração de conhecimentos teóricos e práticos capazes de apoiar a gestão de programas de Pós-Graduação Stricto sensu, em atividades como o fornecimento de informações para o sistema de avaliação da CAPES, análises de produção acadêmica, análises de redes de colaboração de pesquisadores, formulação de políticas, acompanhamento e avaliação de programas de Pós-Graduação, entre outras iniciativas.

Palavras-chave: Pós-Graduação Stricto sensu; Gerenciamento de projetos; Abordagens contingenciais em gestão de projetos; Bancos de dados de produção acadêmica; Ciclo de Vida de Gerenciamento de Projetos.

\footnotetext{
${ }^{1}$ Mestre pela Universidade Nove de Julho - UNINOVE, São Paulo (Brasil)

Analista Judiciário pelo Tribunal Regional Federal da $3^{\mathrm{a}}$ Região - TRF3, São Paulo (Brasil)

E-mail: rcamara62@gmail.com

${ }^{2}$ Doutor pela Universidade de São Paulo - USP, São Paulo (Brasil)

Diretor do Programa de Pós-Graduação em Administração - PPGA da Universidade Nove de Julho UNINOVE, São Paulo (Brasil).

E-mail: maccari@uninove.br

${ }^{3}$ Doutor pela Universidade Federal de São Paulo - UNIFESP, São Paulo (Brasil)

Professor pela Universidade Nove de Julho - UNINOVE, São Paulo (Brasil)

E-mail: renatobio@ hotmail.com
} 


\begin{abstract}
This work consists in an applied, qualitative and exploratory study, carried out by the method of action research. It aims at investigating the contribution of a project management approach to develop a tool with practical application: support Stricto sensu Graduate Program in management. In the initial phase of project, the Adaptive approach was chosen, by applying the Project Management Life Cycle method. The project work includes the integration of data stored in government agencies databases, such as those available in CAPES and CNPq sites. Besides that, includes building a tool prototype, to provide consultations to the integrated data and allows automated generation of entry lists to the program Scriptsucupira. The implementation of proposed tool will facilitate more efficient retrieval of scientific Graduate Brazilian professors production, contributing to generation of knowledge and expertise to support Stricto sensu Graduate Programs management, in activities such as providing information to CAPES evaluation system, academic production analysis, collaborative researchers networks analysis and post-graduation program monitoring and evaluation, among other initiatives.
\end{abstract}

Keywords: Stricto sensu Graduate Programs; Project management; Contingency approaches in project management; Academic production databases; Project Management Life Cycle. 


\section{Introdução}

A forte aceleração no desenvolvimento e uso da informação, iniciou um processo de transição com grandes implicações para as sociedades em todo o mundo, que afetou a educação, entre outros aspectos da vida (UNESCO, 1996). A qualificação do conhecimento como um dos eixos estruturais da atividade produtiva, coloca a educação como condição indispensável para que os países se integrem favoravelmente no processo global de competitividade (Cabral Neto, 2012).

Nesse cenário atuam as Instituições de Ensino Superior (IES), organizações voltadas para a criação, transmissão e disseminação do conhecimento, desempenhando papel destacado nos processos que configuram a sociedade contemporânea (Bernheim \& Chaui, 2003). No Brasil, destacam-se na área os programas de Pós-Graduação Stricto Sensu (PG-SS), conjunto dos cursos de mestrado e/ou doutorado acadêmico e de mestrado profissional (Almeida, 2010; CAPES/MEC, 2010; Moritz, Pereira, Moritz, \& Maccari, 2013; Sidone, 2013).

\subsection{A situação-problema}

A partir da publicação da última edição da Lei de Diretrizes e Bases da Educação (LDB), em 1996, a PG-SS recebeu forte impulso. Houve notável ampliação do acesso ao Ensino Superior e um grande número de egressos, que se traduziu em pressão de demanda pela continuidade da formação, ampliando o número dos cursos de Pós-Graduação. Paralelamente, a PG-SS viu crescer seu valor estratégico, pois a sociedade passou a valorizar mais o papel da educação, associando os Programas à expressão da qualidade da própria IES (Mainardes, Miranda, \& Correia, 2011; Soares, 2014; Teixeira \& Maccari, 2014).

Nesse ambiente competitivo, a gestão eficiente, apoiada por informações atualizadas, confiáveis e disponíveis, tornou-se necessária para equilibrar os custos de atender aos requisitos de qualidade definidos pela Coordenação de Aperfeiçoamento de Pessoal de Nível Superior (CAPES), órgão responsável pela avaliação dos Programas, e ainda enfrentar a concorrência, mantendo uma vantagem competitiva como condição de continuidade (Maccari \& Teixeira, 2014; Meyer Jr., 2008; Soares, 2014).

No Brasil, a referência para obtenção de informações sobre PG-SS são os órgãos governamentais, que disponibilizam na internet dois grandes repositórios: o portal da CAPES e a Plataforma Lattes (PL), do Conselho Nacional de Desenvolvimento Científico e Tecnológico (CNPq) (Guedes, 2001; Moritz et al., 2013). Observa-se, no entanto, uma grande quantidade de informação armazenada em bases de dados heterogêneas. A falta de um acesso unificado dificulta as consultas integradas a documentos e indicadores da atividade científica brasileira (Pacheco \& Kern, 2001; Silva, Maccari, \& Quoniam, 2015).

Por outro lado, existem ferramentas que extraem conhecimento da PL, como o ScriptLattes e sua variante, o ScriptSucupira, cujos recursos computacionais têm produzido conhecimento capaz de apoiar a gestão de Programas de PG-SS. Verifica-se, entretanto, que não é possível, gerar automaticamente listas de entrada para processamento, filtrando informações por critérios que abranjam todo o universo da PG-SS brasileira (Bavaresco, Ferraz, Barro, \& Boeira, 2015; Nigro, Ferraz, Quoniam, \& Alves, 2015; Silva et al., 2015).

Esse cenário sugere os potenciais benefícios do preenchimento dessa lacuna, por meio da criação de uma ferramenta automatizada, a partir daqui chamada de ListaSucupira, capaz de gerar essas listas de entrada, para tornar mais eficiente a obtenção de dados, e dar suporte informacional aos gestores, subsidiando os projetos educacionais voltados à gestão das IES. Analisando as possibilidades de execução desse empreendimento, percebe-se que há pouco 
conhecimento sobre como integrar dados da CAPES e da PL, assim como sobre as características de um artefato dessa natureza, como constataram Silva et al. (2015).

Cleland e Ireland (2006), acreditam que o gerenciamento de projetos é o mecanismo que melhor usa os recursos disponíveis para planejar e implementar produtos que representam uma mudança organizacional, como é o caso da ferramenta ListaSucupira. Nesse sentido, Christopoulos (2004) destaca o fracasso de iniciativas na área de tecnologia, decorrentes da não adoção da estratégia de gestão de projetos como fator crítico de sucesso.

No âmbito da literatura e da prática, há diversas abordagens, já tradicionais, que estabelecem modelos padronizados de aplicação do gerenciamento de projetos. Estudos mais recentes, porém, conferem mais valor ao emprego de técnicas, ferramentas e habilidades adequadas às características de cada projeto. Dessa forma, a adoção de uma abordagem contingencial de gerenciamento, adaptada ao ambiente onde é aplicada, seria mais eficiente para aumentar a chance de sucesso dos projetos nas organizações (Hanisch \& Wald, 2012; L. Junior \& Plonski, 2011; Shenhar \& Dvir, 2010; Wysocki, 2013).

A observação do problema sob essa perspectiva sugere a adesão aos conceitos de gerenciamento de projetos como o ferramental adequado para conduzir o trabalho. A escolha e a aplicação da abordagem e do modelo gerencial seriam, portanto, questões fundamentais para estabelecer a estratégia de condução do projeto, de forma a aumentar a possibilidade de alcançar o sucesso desejado no desenvolvimento da ferramenta ListaSucupira.

\subsection{A questão de pesquisa}

Considerando, dessa forma, o que foi exposto até o presente, este trabalho busca responder à seguinte questão de pesquisa: "Como usar o gerenciamento de projetos para desenvolver uma ferramenta de apoio à gestão de Programas de Pós-Graduação Stricto sensu?".

\subsection{Objetivo}

O objetivo geral da pesquisa é o desenvolvimento de uma ferramenta automatizada, de apoio à gestão de Programas de Pós-Graduação Stricto sensu, baseado na integração dos dados do sítio da CAPES e da Plataforma Lattes, do CNPq.

Para atingir esse objetivo, o trabalho do projeto consiste na criação de uma base de suporte informacional a partir dos dados da CAPES e da Plataforma Lattes; na elaboração de um modelo lógico dos dados integrados; na construção de um protótipo funcional da ferramenta; e na validação do trabalho realizado, avaliando os resultados do processo empregado e da ferramenta desenvolvida. A condução das atividades deve ser direcionada por uma abordagem e um modelo de gerenciamento selecionados para aplicação no projeto.

\section{Referencial Teórico}

Os pilares teóricos considerados relevantes para o desenvolvimento do trabalho compreendem a gestão dos Programas de Pós-Graduação Stricto sensu; o conhecimento sobre produção acadêmica armazenado em bases governamentais; e o gerenciamento do projeto de desenvolvimento da ferramenta. 


\subsection{Gestão da Pós-Graduação Stricto sensu}

O sistema de Pós-Graduação brasileiro foi construído no decorrer das últimas cinco décadas, sendo considerado um dos mais importantes do mundo. Embora tenha se desenvolvido em contexto desfavorável, face ao surgimento tardio das Instituições universitárias, a aplicação de políticas governamentais consistentes lhe permitiu crescer sem perder a qualidade (Balbachevsky, 2005; Neves, Martins, \& Leite, 2002; Santos, 2003).

A CAPES foi criada em 1951, para coordenar a melhoria do nível dos professores universitários e evitar a queda de padrões provocada pela expansão do Ensino Superior. É hoje uma fundação que avalia os Programas de PG-SS; promove a divulgação da produção científica; investe para formar recursos de alto nível; e promove a cooperação científica (CAPES/MEC, 2008; Moritz et al., 2013).

O CNPq, também de 1951, é uma agência voltada à formação de pessoas e ao fomento em pesquisa. Subsidia publicações científicas, apoia a capacitação de pesquisadores, e promove acordos e intercâmbios internacionais. A comunidade científica participa de seus órgãos de gestão e da formulação de sua política institucional (Moritz et al., 2013).

Os esforços despendidos para a gestão dos Programas de PG-SS compreendem a execução de ações e projetos que contemplam, entre outras, atividades de análise de produção acadêmica e de redes de colaboração, programas de pesquisa e desenvolvimento, elaboração de políticas, criação de cursos, definição de currículos, acompanhamento e avaliação de Programas (Campanario, Maccari, Silva, \& Santana, 2009; Silva, Reina, Ensslin, \& Reina, 2012; Ferraz, Quoniam, \& Rezende, 2014; Rolim \& Amaral, 2007).

Outro fundamento da gestão da PG-SS é o cumprimento dos requisitos de qualidade na avaliação da CAPES, maximizando seus indicadores. A avaliação possui caráter imperativo em relação à entrada e permanência dos cursos no mercado. Só são credenciados e têm seus diplomas validados os que obtiverem uma nota satisfatória. Adicionalmente, o acesso aos financiamentos de pesquisas também é vinculado às avaliações da produção acadêmica (Almeida, 2010; CAPES/MEC, 2014a, 2014b; Teixeira \& Maccari, 2014).

\subsection{Conhecimento em bancos de dados de produção acadêmica}

O desenvolvimento da Tecnologia da Informação (TI) no setor público, a procura dos cidadãos por informações, e a necessidade de transparência na gestão, resultaram na crescente disponibilização de dados pelos órgãos governamentais, especialmente os Federais. Em meio aos movimentos recentes ligados à TI, está o desenvolvimento de ferramentas para geração de conhecimento, análise e tomada de decisão a partir desses dados (Junior \& Quintella, 2005).

\subsubsection{Bancos de dados do portal da CAPES}

No processo de avaliação da CAPES, os responsáveis pelos Programas informavam uma série de dados relacionados ao período de avaliação dos cursos por meio de um sistema informatizado outrora denominado Coleta Capes, específico para esse objetivo (Akim, Mergulhão, \& Borrás, 2013). A partir de 2014, o sistema foi integrado à Plataforma Sucupira (PS), ferramenta que disponibiliza informações, processos e procedimentos em tempo real (CAPES/MEC, 2014c).

Os dados das avaliações dos Pogramas de PG-SS são agrupados pelo sistema em onze documentos temáticos, organizados para a emissão dos Cadernos de Indicadores, os relatórios 
usados no processo de avaliação propriamente dito. Para garantir a transparência e disseminar o conhecimento sobre sua avaliação, a CAPES disponibiliza o acesso aos relatórios produzidos, divulgando os resultados em seu portal da internet e disponibilizando os cadernos para download (CAPES/MEC, 2014a, 2014c).

Outros dados estatísticos sobre a PG-SS se encontram disponíveis na ferramenta Geocapes, um aplicativo que exibe o conteúdo de nove bases de dados, de acordo com sua localização geográfica, em modo gráfico ou de planilha. As informações relativas ao Brasil estão consolidadas por estado, e detalhadas por município. As informações do exterior estão agregadas por país (CAPES/MEC, 2014d; MEC, 2008).

\subsubsection{Plataforma Lattes e currículos Lattes}

A Plataforma Lattes compreende um conjunto de sistemas computacionais desenvolvido e mantido pelo $\mathrm{CNPq}$, para gerenciar suas atividades de fomento e integrar informações sobre pesquisadores e instituições, brasileiros e estrangeiros (Guedes, 2001). O principal módulo da PLé o dos currículos Lattes, sistema de informações curriculares onde estão armazenados dados pessoais e profissionais, produção acadêmica e tecnológica, de milhares de instituições e milhões de docentes, alunos e outros profissionais de ciência, tecnologia e inovação (Alves, Yanasse, \& Soma, 2012).

Os currículos são disponibilizados em formato Hyper Text Markup Language (HTML), constituindo importante fonte de informação sobre produções acadêmicas. Podem ser destacadas outras facilidades da plataforma, como o uso exclusivo pela internet; a configuração personalizada de relatórios; a compatibilidade com diversos editores de texto; a identificação de inconsistências; a verificação do preenchimento de campos obrigatórios; o acesso direto à página do grupo de pesquisa; o perfil da produção cientifica do usuário; e a identificação de possíveis duplicidades (Ferraz, Quoniam, \& Maccari, 2014; Guedes, 2001).

Em contrapartida, existem dificuldades para o pleno uso da PL. Uma das principais referese à pesquisa da produção de grupos, pois os currículos Lattes são disponibilizados de forma individualizada. Adicionalmente, o acesso aos currículos cadastrados não é livre, e quem se propõe a pesquisar dados para estudos macro fica na dependência da liberação de acesso por parte do CNPq (Quoniam \& Ferraz, 2014).

\subsubsection{A ferramenta ScriptSucupira, para extração de dados da Plataforma Lattes}

O ScriptLattes e sua variante, o ScriptSucupira, são ferramentas de software livre, disponíveis para extração e compilação automáticas de informações sobre conjuntos de pesquisadores. Disponibilizam em uma página web produções bibliográficas, técnicas e artísticas, orientações, projetos de pesquisa, prêmios e títulos, grafos de colaboração e mapas de geolocalização. Seus relatórios e gráficos refletem as informações extraídas no momento da execução (Maccari, 2015; Mena-Chalco \& Junior, 2011; Nigro et al., 2015).

O sistema ScriptLattes/ScriptSucupira, a partir daqui chamado apenas de ScriptSucupira, é composto por seis módulos com funções específicas. Os três primeiros, seleção, pré-processamento e tratamento de redundâncias, tratam da recuperação e tratamento dos dados. Os ouros três, geração de grafos de colaboração, geração de mapas de pesquisa e criação de relatórios, relacionam-se aos artefatos que pode produzir (Nigro et al., 2015).

No âmbito da gestão dos Programas de PG-SS, destaca-se a agilidade da ferramenta na coleta dos dados. O acompanhamento da produção acadêmica torna-se mais simples e eficiente. 
Os gestores analisam o desempenho dos docentes em suas linhas de pesquisa, identificando e corrigindo antecipadamente deficiências, para garantir bons resultados na avaliação (Bavaresco et al., 2015; Maccari, 2015; Nigro et al., 2015).

\subsection{Projetos e gerenciamento de projetos}

As diversas definições de "projeto" encontradas na literatura convergem no sentido de considerá-los como conjuntos de atividades interdependentes, iniciativas temporárias, singulares, multifuncionais, que consomem recursos limitados, orientadas para um objetivo de mudança, empreendidas para criar um produto, serviço ou resultado específico (Cleland \& Ireland, 2007; Kerzner, 2011; Maximiano, 2014; PMI, 2013; Shenhar \& Dvir, 2010; Wysocki, 2013). Expandindo o conceito, Shenhar (2012) e Wysocki (2013) consideram que o foco principal, que valida a realização do projeto, deve estar na criação e entrega de valor de negócio, de vantagem competitiva e fortalecimento da organização no mercado.

As abordagens tradicionais de gerenciamento de projetos aplicam práticas padronizadas para definir os processos de planejamento, organização e controle da equipe (Cleland \& Ireland, 2007; Williams, 2005). Segundo essa visão, os projetos são essencialmente similares entre si, e a aplicação adequada do corpo convencional de conhecimentos é suficiente para conduzi-los a resultados positivos (Shenhar \& Dvir, 2010).

As abordagens contingenciais, por sua vez, tratam os projetos como diferentes entre si. Procuram identificar as características, necessidades e critérios de sucesso aplicáveis aos diferentes tipos. Defendem que o gerenciamento do projeto deve ser adequado ao conteúdo, ao ambiente em que se desenvolve, e às características específicas da organização (Carvalho \& Rabechini Jr., 2011; Fernandez \& Fernandez, 2008; Hanisch \& Wald, 2012; Wysocki, 2013).

\subsection{Categorização de projetos}

Wysocki (2013), credita parte das falhas em projetos à inexistência de uma maneira prática e eficaz de adequar as abordagens de gerenciamento aos tipos de projetos conduzidos no ambiente de negócios atual. Nesse contexto, Chagas Jr, Perondi e Yassuda (2013), concluem que a diversidade de tipos evidencia a importância de categorizar os projetos, por meio de um sistema que permita identificar variáveis e subgrupos com características comuns de gerenciamento, aplicáveis de forma suficientemente genérica para que se possa propor a melhor sistemática de gestão a ser implementada.

Considerando como principais critérios para categorização de projetos a incerteza existente sobre seus objetivos e soluções, Wysocki (2013) desenvolveu o modelo dos Ciclos de Vida do Gerenciamento de Projetos (CVGP), uma tipologia que classifica o projeto e recomenda para ele uma abordagem de gerenciamento. Nesse modelo, objetivos e soluções são representados em um eixo e quantificados em duas métricas objetivas: claro ou não e completo ou não.

Dependendo de suas características um projeto é classificado como i) Tradicional, onde objetivos e soluções são claros, no quadrante 1; ii) Ágil, onde os objetivos são claros, mas as soluções não, no quadrante 2; iii) Extremo, onde nem objetivos nem soluções são claros, no quadrante 3; e iv) Omertxe, onde as soluções são claras, mas os objetivos não, no quadrante 4 (Marinho, Sampaio, Lima, \& de Moura, 2014; Wysocki, 2013). Segundo os autores, essa representação expressa a visão dos cenários possíveis de um projeto, em uma matriz de quatro quadrantes, mostrado na Figura 1: 


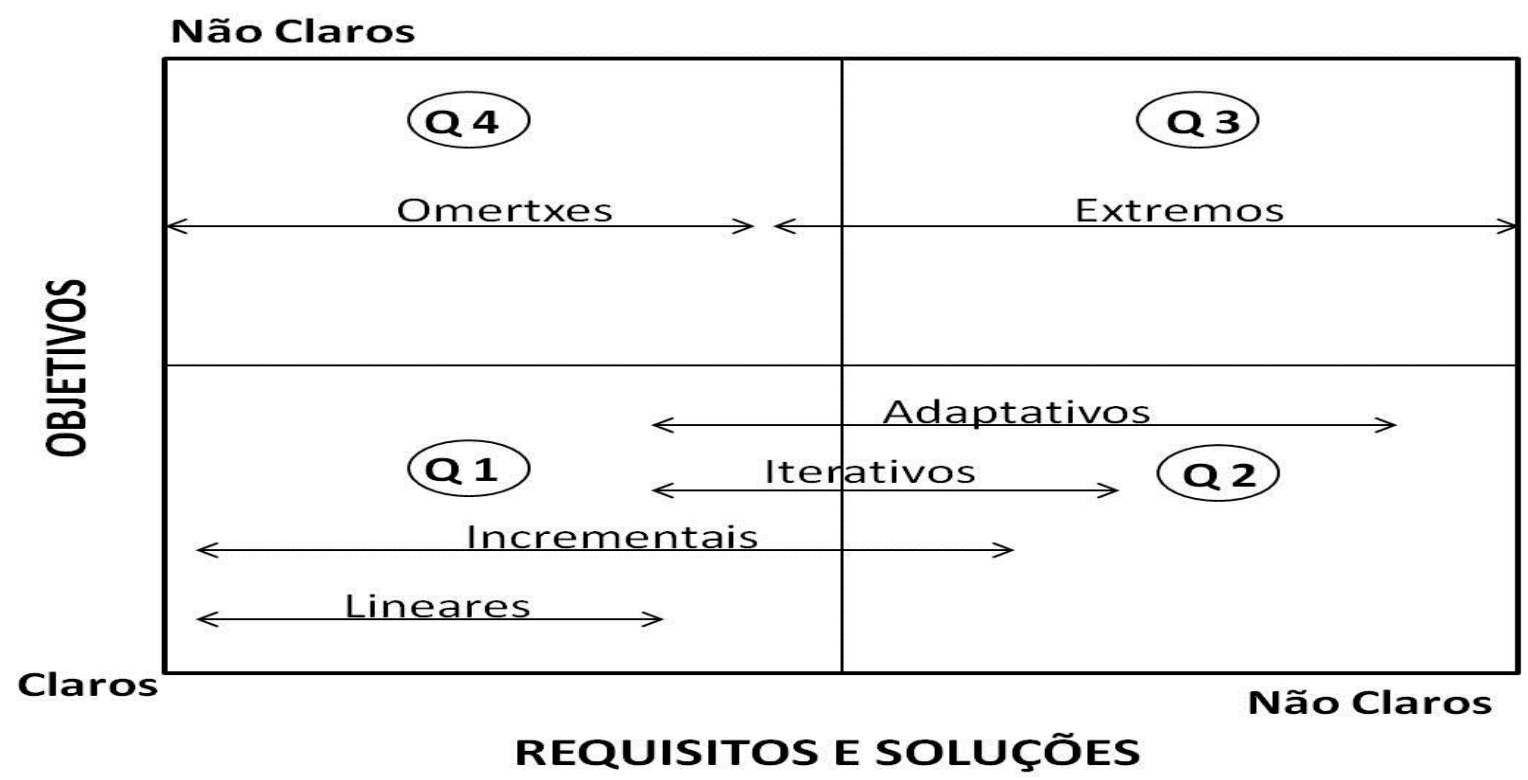

Figura 1. Representação da matriz do Ciclo de Vida do Gerenciamento de Projetos Fonte: Adaptado de Wysocki (2013, p. 118)

O modelo CVGP identifica cinco diferentes ciclos de vida, relacionados aos tipos de projeto: Linear e Incremental, para o tipo Tradicional (Q1); Iterativo e Adaptativo, para o tipo Ágil (Q2); Extremo (Q3) e Omertxe (Q4), para o tipo Extremo. Os dois últimos são considerados iguais e opostos, tanto que Omertxe corresponde a Extremo, lido de trás para frente (Marinho et al., 2014; Wysocki, 2013).

\section{Metodologia}

Em conformidade com o protocolo proposto por Biancolino, Kniess, Maccari, \& Rabechini Jr. (2012), este relato técnico intenciona compartilhar uma experiência de natureza técnica e aplicação prática. Foi elaborado buscando conferir às suas atividades, o rigor científico e metodológico próprio das produções tecnológicas.

O propósito do trabalho foi a geração de conhecimentos e elaboração de artefatos aplicáveis na prática, contribuindo ao mesmo tempo para a solução de um problema específico em uma organização. Essa abordagem se enquadra no conceito de pesquisa aplicada, de acordo com a classificação proposta por Gerhardt e Silveira (2009).

Como iniciativa de caráter científico, propõe-se investigar a realidade, entender fatos e fenômenos para construir a solução de um problema observado, enquanto produz novos conhecimentos (Gil, 2008; Theóphilo \& Martins, 2009). Os elementos observados são considerados da maneira como aparecem, procurando o pesquisador apenas compreendê-los e descrevê-los, sem explicar seus motivos (Gil, 2010).

Empregou-se a abordagem qualitativa, caracterizada por não medir eventos nem fazer análises estatísticas. Os dados são obtidos no contato direto com a situação e analisados sob diferentes perspectivas, apoiando-se na experiência dos pesquisadores para refletir e tomar decisões durante o processo de produção do conhecimento (Flick, 2009; Godoy, 1995). 
A estratégia de pesquisa adotada foi o método da Pesquisa-Ação (PA), uma investigação onde os participantes cooperam para entender seu ambiente, identificar problemas e buscar soluções para eles. A produção e o uso do conhecimento acontecem simultaneamente. A estrutura geral do processo seguiu a proposta de autores que preconizam sua divisão em quatro fases principais: exploratória, pesquisa aprofundada, ação e avaliação. Na prática, entretanto A execução das duas fases intermediárias acontece de forma simultânea (Gil, 2008; Theóphilo \& Martins, 2009; Thiollent, 2009, 2011).

$\mathrm{Na}$ fase exploratória, as atividades desenvolvidas foram a definição da unidade de análise e a delimitação das áreas envolvidas; a definição dos participantes; a investigação do problema, utilizando fontes primárias e secundárias diversas; e a elaboração de uma proposta para a realização do trabalho.

A fase de pesquisa aprofundada consistiu em planejar e detalhar o desenvolvimento do trabalho, incluindo a escolha da abordagem de gerenciamento, a definição da integração dos dados e a especificação de requisitos dos artefatos.

A fase de ação correspondeu às atividades das quatro etapas necessárias para integrar os dados, elaborar o modelo lógico e construir o protótipo da ferramenta, aplicando no processo os métodos de gerenciamento definidos.

$\mathrm{Na}$ última fase foram avaliados o desenvolvimento do trabalho, as atividades e os resultados, considerando o referencial teórico que sustentou o desenvolvimento da pesquisa.

\section{Apresentação e análise dos resultados obtidos}

Apresenta-se a seguir o detalhamento das atividades desenvolvidas, abrangendo as fases da pesquisa-ação, à luz do referencial teórico, seguindo a sequência lógica definida em sua estrutura: exploratória, de pesquisa aprofundada, de ação e de avaliação.

\subsection{Considerações sobre a fase exploratória}

Como unidade de análise, foi escolhido um Programa de PG-SS em Administração, de uma universidade localizada na cidade de São Paulo - SP, com facilidade de acesso às informações, disponibilidade dos participantes e interesse pela pesquisa. Um único pesquisador ficou responsável pelo trabalho. O coordenador do Programa recebeu o papel de usuário-gestor, responsável pelas informações sobre o Programa e suas necessidades. Com relação ao ScriptSucupira, garantiu-se o acesso do pesquisador ao grupo que trabalha com a ferramenta.

$\mathrm{Na}$ pesquisa bibliográfica, foram consultadas referências publicadas em livros, periódicos, revistas, jornais, sítios na internet e anais de congressos, entre outros. Adicionalmente, foram realizadas entrevistas com os pesquisadores do tema e os representantes da Instituição pesquisada. As entrevistas foram abertas e não foram gravadas. Foram realizadas anotações em papel, e o material digital recebido foi gravado em um pendrive.

As entrevistas com o grupo de pesquisa se destinaram a adquirir conhecimento. Foram repassadas informações sobre os dados disponíveis no portal da CAPES e na Plataforma Lattes. Foram também disponibilizadas rotinas para download dos cadernos de avaliação e recuperação dos identificadores de acesso aos currículos, desenvolvidas em linguagem Phyton de programação. Confirmou-se ainda a inexistência de ferramentas semelhantes à proposta neste trabalho.

Nas entrevistas com o coordenador do Programa, discutiram-se as necessidades informacionais de gestão. Destacaram-se aquelas sobre produção acadêmica dos docentes e 
suas redes de colaboração, bem como sobre Programas da mesma área em outras IESs. Também decidiu-se que os dados abrangeriam apenas os Programas de PG-SS do Estado de São Paulo, da área de Administração, Ciências Contábeis e Turismo, lavando-se em conta os dados oriundos da avaliação realizada em 2012.

Na proposta de trabalho elaborada, mostrada na Tabela 1, optou-se por sugerir uma ferramenta estruturada em dois módulos: uma base de dados sobre PG-SS, e uma aplicação informatizada. Para este estudo, além da integração dos dados, propõe-se a elaboração de dois artefatos: um modelo dos dados integrados e um protótipo funcional da ferramenta.

\section{Tabela 1}

\section{Etapas de execução do trabalho, atividades e entregas}

\begin{tabular}{|c|c|c|c|}
\hline Macroetapas & Etapas & Atividades & Entregas \\
\hline \multirow{2}{*}{$\begin{array}{l}\text { Integração } \\
\text { dos dados }\end{array}$} & $\begin{array}{l}\text { Tratamento das } \\
\text { informações da } \\
\text { CAPES }\end{array}$ & $\begin{array}{l}\text { Consultas manuais ao Geocapes } \\
\text { Consultas automatizadas aos cadernos } \\
\text { de avaliação } \\
\text { Tratamento dos dados }\end{array}$ & $\begin{array}{l}\text { Lista consolidada de IESs e } \\
\text { programas } \\
\text { Lista consolidada de } \\
\text { docentes }\end{array}$ \\
\hline & $\begin{array}{l}\text { Tratamento das } \\
\text { informações da } \\
\text { Plataforma Lattes }\end{array}$ & $\begin{array}{l}\text { Consultas manuais e automatizadas aos } \\
\text { currículos Lattes } \\
\text { Tratamento dos dados }\end{array}$ & $\begin{array}{l}\text { Lista consolidada de } \\
\text { docentes, acrescida dos } \\
\text { dados da Plataforma Lattes }\end{array}$ \\
\hline \multirow{2}{*}{$\begin{array}{l}\text { Elaboração } \\
\text { dos artefatos }\end{array}$} & $\begin{array}{l}\text { Elaboração do } \\
\text { modelo lógico } \\
\text { dos dados }\end{array}$ & $\begin{array}{l}\text { Análise das listas consolidadas } \\
\text { Análise dos requisitos } \\
\text { Normalização e modelagem dos dados }\end{array}$ & Modelo lógico dos dados \\
\hline & $\begin{array}{l}\text { Elaboração do } \\
\text { protótipo da } \\
\text { ferramenta }\end{array}$ & $\begin{array}{l}\text { Análise das listas consolidadas } \\
\text { Análise dos requisitos } \\
\text { Elaboração do protótipo }\end{array}$ & $\begin{array}{l}\text { Protótipo da aplicação em } \\
\text { funcionamento, }\end{array}$ \\
\hline
\end{tabular}

Nota. Elaborada pelos autores

Os principais pontos da proposta de trabalho foram então registrados em uma Ficha de Projeto, submetida à apreciação dos usuários, para subsidiar a homologação da fase exploratória. A proposta foi aceita e o seu prosseguimento foi então autorizado.

\subsection{Considerações sobre a fase de pesquisa aprofundada e a fase de ação}

O modelo CVGP, de Wysocki (2013), foi adotado para categorizar o projeto e selecionar a abordagem de gerenciamento adequada. O processo de seleção iniciou-se pela identificação dos requisitos de alto nível do projeto, voltados para os objetivos de negócio. A análise do resultado evidenciou um alto grau de incerteza. Isto posicionou o projeto no quadrante 2 , caracterizado pela pouca clareza da solução. A abordagem escolhida, então, foi a do ciclo Adaptativo, com foco na descoberta gradativa da solução (Wysocki, 2013).

A seguir, procedeu-se à análise do impactos dos fatores de risco e à adequação da estrutura do ciclo de vida Adaptativo à proposta de trabalho elaborado para o projeto. A Figura 2 representa a estrutura adaptada resultante. 


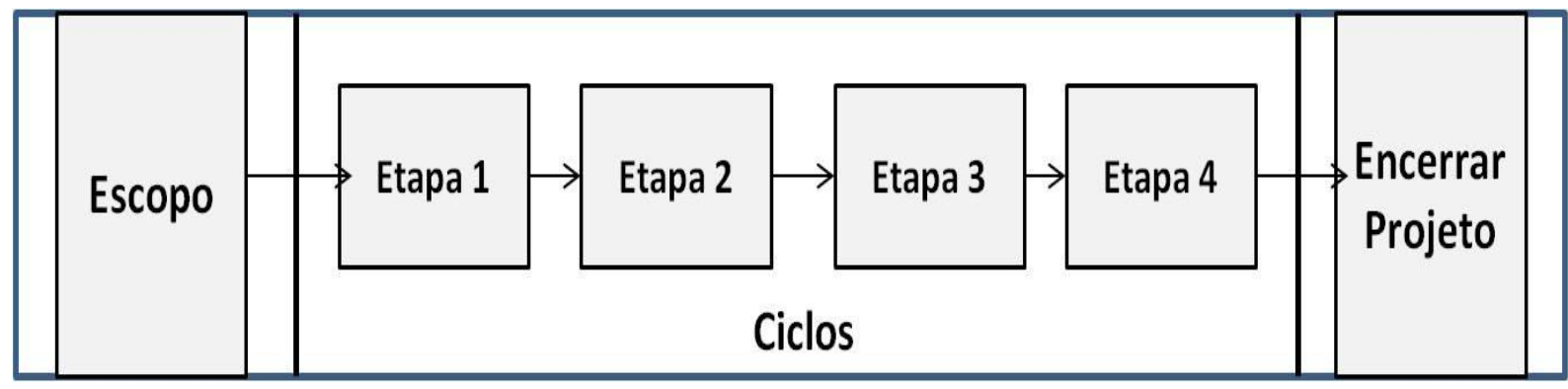

Figura 2. Adequação da estrutura do ciclo de vida Adaptativo ao projeto Fonte: Elaborada pelos autores

A fase inicial corresponde ao detalhamento do escopo. A ela, seguem-se as iterações, chamadas de Ciclos, que destinam-se à construção de elementos já definidos e à descoberta de novos. A fase de ciclos, apenas uma no modelo, correspondeu às quatro etapas do plano de trabalho. Cada etapa engloba a sequência completa de um ciclo: planejar, iniciar, monitorar, controlar, encerrar e avaliar. No planejamento das etapas, os requisitos são gradualmente identificados e registrados. A parte final correspondente ao encerramento do projeto.

$\mathrm{Na}$ fase de detalhamento do escopo, foram especificados os dois módulos da ferramenta: a base de dados e a aplicação. Definiu-se que, na base, ficam armazenados os dados integrados, referentes às IESs, aos Programas e docentes. Optou-se por uma base própria devido à dificuldade de recuperar estes dados nas bases originais a cada consulta solicitada. A aplicação proposta tem baixa/média complexidade. Deve prever a geração das listas de entrada, as consultas e a manutenção dos dados, bem como garantir a consistência dos critérios selecionados. O passo seguinte foi o desenvolvimento das etapas do trabalho.

\subsubsection{Etapa 1 - Tratamento dos dados do portal da CAPES}

A entrega produzida para essa etapa foi uma planilha, contendo os dados consolidados de IES, Programas e docentes. Esse material foi elaborado por meio de consultas manuais às páginas da ferramenta Geocapes (http://geocapes.capes.gov.br/geocapes2/), download automatizado dos cadernos da avaliação de 2010-2012, extração e validação de dados dos docentes neles contidos.

Cabe destacar os esforços de aquisição e aprofundamento do conhecimento empreendidos nessa atividade, conforme o modelo Adaptativo (Wysocki, 2013). Os mais relevantes foram de ordem técnica, com respeito à absorção das características da versão 3 da linguagem de programação Python (https://www.python.org/), e à escolha da ferramenta PDFMiner (http://www.unixuser.org/ euske/python/pdfminer/), para conversão de arquivos PDF para o formato TXT.

O processo de extração dos dados dos docentes visando fornecer um banco de dados para atuação do ListaSucupira surgiu no decorrer do trabalho. As características específicas do projeto precisaram ser consideradas, pelo fato da estrutura dos arquivos não seguir uma lei de formação conhecida. Os ciclos de extração e análise identificaram particularidades que levaram ao entendimento do posicionamento dos dados nos arquivos. As regras descobertas foram codificadas em scripts específicos, processados em ciclos para a extração automatizada. A cada ciclo, novas características dos dados foram descobertas e as soluções para extraí-los progressivamente incorporadas ao código.

Ao final de um ciclo avaliava-se o prosseguimento do processo, como previsto no modelo, comparando-se os resultados do processamento com os dados originais. Um procedimento 
automatizado final comparou as quantidades recuperadas dos cadernos com as publicadas no portal da CAPES. Uma vez que não houve divergências, os resultados produzidos na etapa foram considerados corretos.

Concluídos os ciclos desta etapa, os dados consolidados das IESs e Programas, além da lista de 526 docentes, foram exportados para uma planilha Excel. Cada conjunto de informações foi organizado em uma aba. A planilha ficou armazenada como produto final da etapa e como evidência da corretude dos dados, possibilitando a passagem para a etapa seguinte.

\subsubsection{Etapa 2 - Tratamento dos dados da Plataforma Lattes}

Os dados da Plataforma Lattes foram tratados e recuperados por meio de consultas automatizadas, complementadas por buscas manuais e consultas na internet. Foram acrescentados à lista de docentes os identificadores de acesso aos currículos, chamados aqui de idLattes 10 e idLattes 16, bem como os resumos curriculares dos docentes. O resultado foi então validado e gerou-se uma lista consolidada de docentes, em formato de planilha. Os "id" ou identificadores correspondem a um registro de 10 ou de 16 dígitos que dão acesso a uma página da internet onde o currículo de cada pesquisador está armazenado (Nigro, Ferraz, Quoniam, Alves, $\&$ Messais, 2016).

A produção de conhecimento aconteceu com relação à recuperação dos dados nas páginas HTML de resposta, ao refinamento das estratégias de busca de nomes, à definição dos critérios de rankeamento, e à seleção dos algoritmos de verificação de similaridade.

Ciclos de processamentos e análises permitiram descobrir como recuperar os IdLattes16 e os resumos curriculares na Plataforma Lattes sem precisar resolver o captcha, recurso computacional usado para dificultar buscas automatizadas inserido na Plataforma Lattes pelo CNPq. O método descoberto, porém, só se aplica às respostas onde existem links para a rede de colaboração do docente ou para os diretórios dos seus grupos de pesquisa. Onde não existe, foi necessário fazer consultas manuais para encontrar as informações desejadas.

Para validação dos dados, definiu-se um processo automatizado de classificação, ou rankeamento, pontuando as correspondências entre os dados recuperados dos currículos e da CAPES. Para o nome, comparou-se o caderno de avaliação com o currículo Lattes, usando os algoritmos da Distância de Levenshtein e o Coeficiente de Dice para calcular a similaridade.

$\mathrm{Na}$ análise do rankeamento, verificou-se que a pontuação alcançou $72,42 \%$ do máximo possível, para os nomes com IdLattes 16 único, e de 70,65\% para os docentes com múltiplos IdLattes16. Esses índices foram considerados suficientes para oferecer boa margem de segurança às análises dos gestores. Para aumentar a confiabilidade, foi conferida a correspondência entre o nome presente no caderno e nome registrado no currículo encontrado, constatando-se um percentual de acerto de $100 \%$.

Com base nessa validação, foram considerados corretos os resultados produzidos na etapa. Os dados foram organizados em duas abas de uma planilha de resultados. Essa planilha também ficou armazenada como produto final da etapa, servindo como registro do histórico de pontuação e evidência da corretude dos dados gerados, bem como representando a conclusão da integração dos dados. 


\subsubsection{Etapa 3 - Análise e elaboração do modelo lógico dos dados}

Os pré-requisitos para essa etapa foram a existência do conjunto de dados integrados e o conhecimento adquirido sobre eles, ocorridos nas etapas anteriores. O trabalho, essencialmente técnico, foi realizado pelo pesquisador, com base em seus conhecimentos e sua experiência, conforme Flick (2009) e Thiollent (2011) preveem ser possível numa PA.

Inicialmente foram identificados os principais grupos de elementos de informação, seus atributos, a indicação de sua origem e dos valores que podem assumir. Foram contemplados dados de docentes, programas, avaliações e IES, complementadas por informações de apoio, para atender aos requisitos dos usuários, facilitar a implementação e a manutenção, e criar um modelo flexível para futuras expansões. Durante a criação do modelo, outros dados foram acrescentados aos já identificados.

A forma de armazenamento escolhida foi o banco de dados do modelo relacional, a tecnologia mais utilizada no mercado. Para apoiar a elaboração do modelo, usou-se a ferramenta de modelagem brModelo (http://sis4.com/brModelo/download.aspx). Na execução da modelagem, a técnica de normalização foi aplicada sobre os dados para diminuir a redundância e as chances de criar inconsistências.

O modelo desenvolvido compreende 19 tabelas e 82 campos. Nele estão indicados os relacionamentos lógicos e a integridade referencial, de acordo com os princípios teóricos do modelo relacional. Os tipos de dados são genéricos, apenas indicados como "Número", "Texto" e "Booleano", sem especificação de tamanho.

\subsubsection{Etapa 4 - Construção do protótipo da ferramenta}

A última etapa consistiu na construção do protótipo da ferramenta, a partir da planilha de resultados produzida na etapa de tratamento dos dados da Plataforma Lattes. Assim como na etapa anterior, as atividades também foram de natureza técnica, executadas em sua maior parte pelo pesquisador, apoiado em seus conhecimentos e sua experiência.

Para atender às necessidades identificadas junto ao usuário gestor, ficou definido que o protótipo deveria contemplar três funcionalidades básicas: i) permitir consultas, para a formação dos grupos de docentes a partir de diversos critérios; ii) selecionar os dados que atendem aos critérios definidos; e iii) gerar a lista de docentes para a execução do ScriptSucupira.

A planilha Excel foi escolhida para ser o suporte do protótipo, por manter a compatibilidade com o restante do trabalho, atender nativamente às funcionalidades de seleção e consulta de dados, e permitir o desenvolvimento de rotinas para a geração das listas, por meio da linguagem Visual Basic for Applications (VBA).

O protótipo elaborado consiste de uma planilha com duas abas, sendo a primeira para selecionar dados, e a segunda para gerar listas de docentes. Para utilizar, basta copiá-la para qualquer pasta onde o Excel esteja instalado. A Figura 3 mostra a primeira aba do protótipo. 


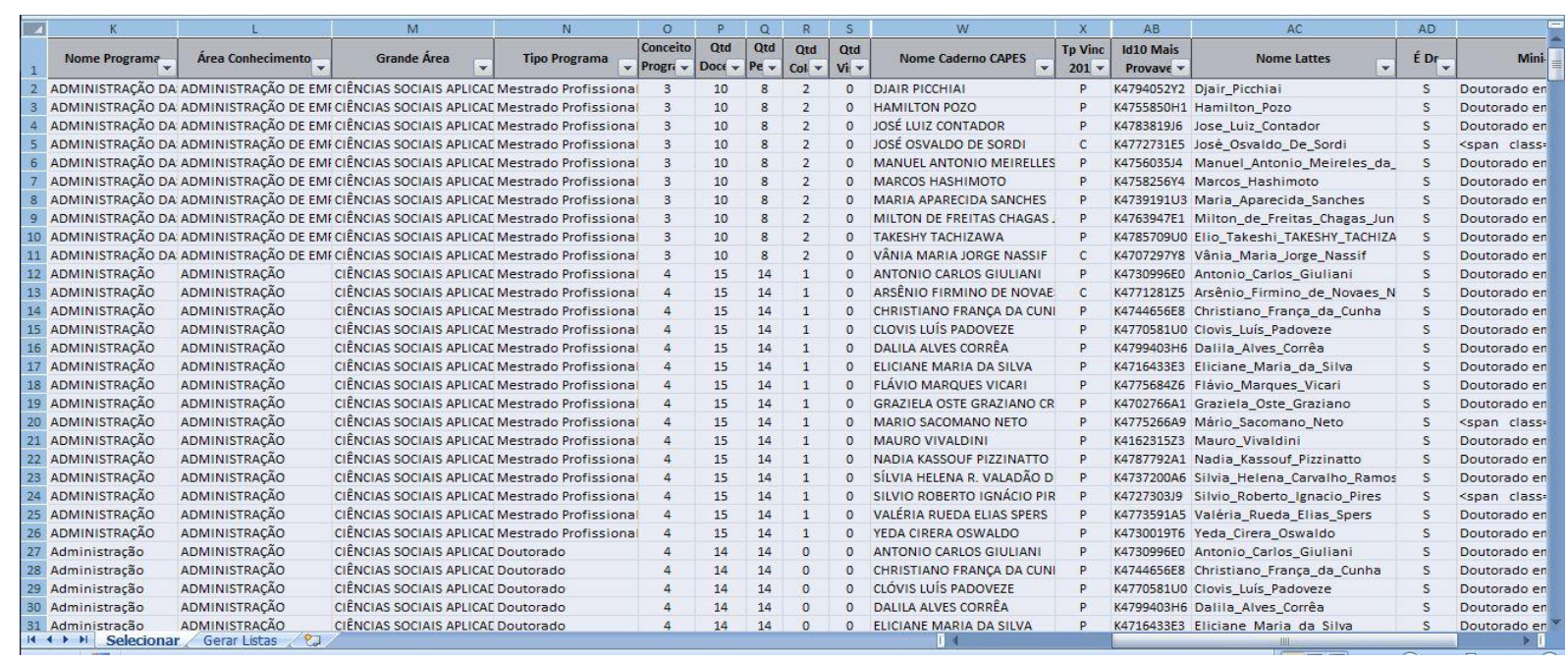

Figura 3. Aba de seleção de dados do protótipo Fonte: Elaborada pelos autores

O primeiro passo para gerar uma lista de docentes é a seleção dos nomes. Os grupos podem ser formados a partir de diversos critérios. Sobre os dados, podem ser aplicados os filtros disponibilizados pela planilha Excel, possibilitando uma grande variedade de escolhas: autofiltros, filtros de número e texto, e filtros avançados. A cada critério, são exibidos apenas os dados que atendem a ele, compatíveis com os dados até então selecionados para a mesma consulta. Esse recurso previne a ocorrência de erros e garante a integridade das opções.

Depois de selecionar o grupo de docentes de sua lista, o usuário comanda o procedimento de geração das listas para o ScriptSucupira, a partir da segunda aba. Todas as funcionalidades foram programadas pelo pesquisador, usando a linguagem VBA. A aba compreende uma área de exibição de dados e quatro botões. A Figura 4 mostra seu aspecto.

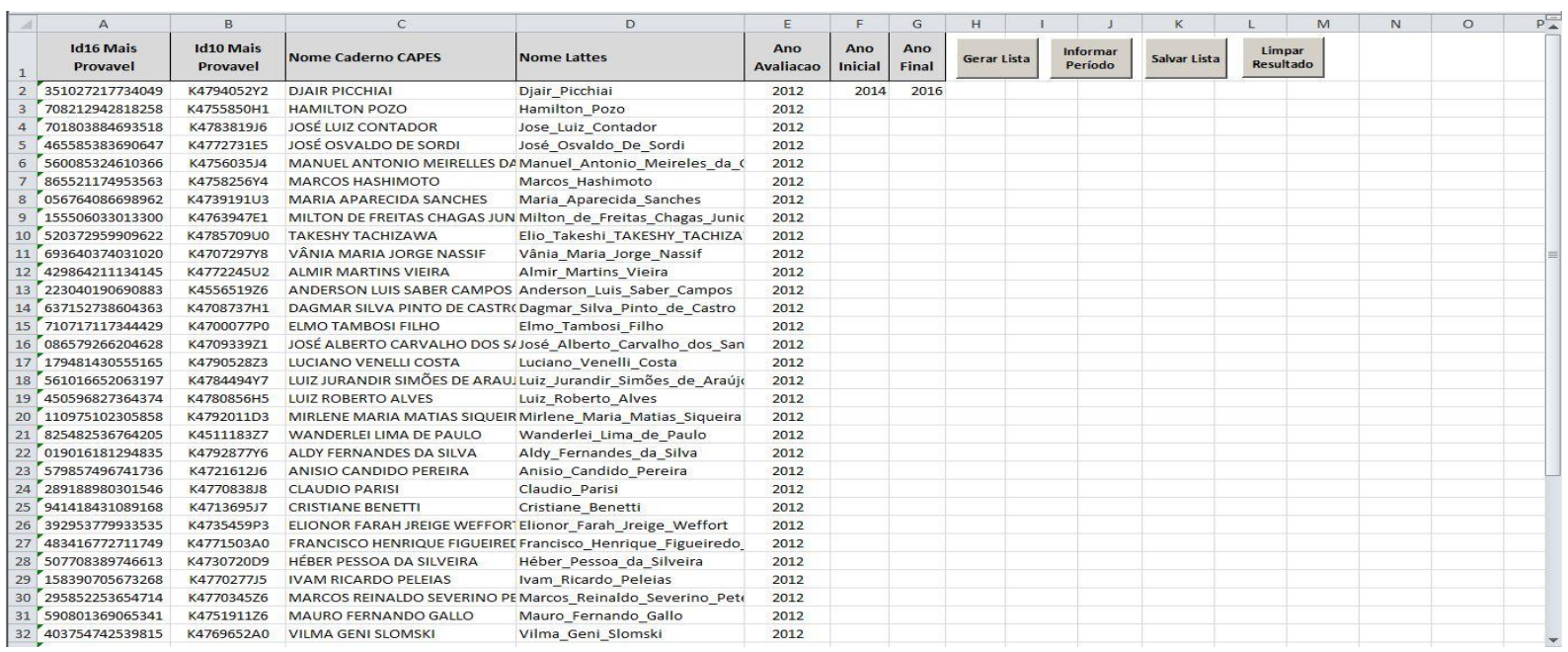

Figura 4. Aba de geração de listas de nomes do protótipo Fonte: Elaborada pelos autores

O botão "Gerar Lista" transfere para a área de dados as colunas específicas das linhas selecionadas na primeira aba. Os dados só são trazidos se a área de exibição estiver vazia, por isso, as linhas em exibição, referentes à última consulta realizada, precisam ser apagadas. Para isso, deve-se acionar o botão "Limpar Resultado". 
O botão "Salvar Lista" grava em um arquivo do tipo texto os dados da área de exibição, menos o cabeçalho. O arquivo é armazenado na mesma pasta em que se encontra a planilha do protótipo. Antes de solicitar a gravação, é preciso informar o período a ser considerado para processamento, acionando-se o botão "Informar Período".

A validação da aderência aos requisitos de negócio foi feita pelo usuário-gestor. As funcionalidades do protótipo foram apresentadas, junto às instruções de funcionamento. $\mathrm{O}$ usuário apresentou sugestões relacionadas à nomenclatura e à visibilidade dos campos, e aprovou o produto entregue, validando as funcionalidades implementadas.

\subsection{5 $\mathrm{O}$ encerramento do projeto e das fases de pesquisa aprofundada e de ação}

Um projeto executado segundo o modelo Adaptativo termina quando o prazo ou o orçamento são excedidos, o projeto é cancelado, ou uma solução aceitável é encontrada. Neste caso, o encerramento deu-se pelo último critério, após a conclusão da fase de ciclos e a aceitação dos resultados, o que também encerrou as fases intermediárias da PA.

Deve-se destacar que o modelo Adaptativo também preconiza a satisfação dos requisitos de alto nível definidos para o projeto. Sob essa perspectiva, considerou-se alcançado o nível desejado de sucesso. Todos os artefatos produzidos, bem como o conjunto da solução encontrada, foram homologados e aceitos pelo usuário gestor.

\subsection{Considerações sobre a fase de avaliação}

A análise realizada para definição da abordagem de gerenciamento, ainda nas fases iniciais, identificou o desafio central do projeto, evidenciando o benefício trazido pela iniciativa. Nesse mesmo processo, a elicitação dos requisitos de alto nível evitou análises demoradas de procedimentos pouco conhecidos e estabeleceu um foco para o trabalho, possibilitando a identificação e correção de desvios, ao fim de cada ciclo.

$\mathrm{Na}$ execução das etapas do trabalho, o modelo Adaptativo de gerenciamento mostrou-se ajustado às necessidades, especialmente aos problemas decorrentes da incerteza. Os sucessivos ciclos de planejamento, execução e avaliação revelaram-se eficientes. Como previsto, aplicar o conhecimento de um ciclo no planejamento do ciclo seguinte conduziu à descoberta gradativa da solução para o problema.

Nas avaliações de encerramento de ciclo, as necessidades de novos conhecimentos e tecnologias foram identificadas com antecipação. A flexibilidade no controle do prazo e na definição do escopo facilitaram a incorporação de novos elementos ao projeto, possibilitando a gestão mais ágil e eficiente das mudanças e dos fatores imprevistos.

Ao final, além dos produtos entregues, restaram como benefícios residuais o conhecimento sobre linguagens de programação Python e VBA, integração dos dados, consultas aos currículos Lattes e extração de dados de arquivos PDF.

\section{Conclusões e considerações finais}

Esta pesquisa foi conduzida em uma IES, para investigar a aplicação de uma abordagem contingencial no gerenciamento de um projeto para desenvolver uma ferramenta de apoio à gestão de PG-SS. Tal artefato possibilitará a consulta de dados integrados do portal da CAPES e da Plataforma Lattes, além da geração automatizada de listas de entrada, para processamento 
pelo programa ScriptSucupira. O modelo Adaptativo de gerenciamento foi o escolhido, fundamentado numa abordagem contingencial, pelo método do Ciclo de Vida do Gerenciamento de Projetos, de Wysocki (2013).

A aplicação dos preceitos do modelo Adaptativo mostrou-se fundamental para encontrar uma solução para o problema do desenvolvimento da ferramenta. As características contingenciais de adaptabilidade e flexibilidade do modelo adequaram-se ao enfrentamento da principal dificuldade do projeto, a incerteza relacionada aos procedimentos e requisitos para integrar os dados e construir o protótipo da ferramenta.

Os critérios de sucesso previstos relacionavam-se aos objetivos de negócio, a serem atendidos pelos resultados alcançados. Nesse sentido, os representantes da Instituição pesquisada homologaram positivamente os artefatos resultantes do projeto. A produção, entrega e homologação desses artefatos também determinaram o sucesso no cumprimento dos demais objetivos previstos.

Conclui-se, dessa forma, que as diversas atividades e análises levadas a efeito durante o trabalho, responderam de forma afirmativa à questão de pesquisa proposta. Os resultados mostraram que há maneiras possíveis de aplicar uma abordagem contingencial de gerenciamento de projetos para desenvolver uma ferramenta de apoio à gestão de programas de PG-SS. Adicionalmente, indicaram que a aplicação dessa abordagem facilita o desenvolvimento, em especial na gestão das mudanças inerentes às incertezas do ambiente.

Acredita-se que a ferramenta apresentada preenche uma lacuna nas análises de produção acadêmica voltadas à gestão de PG-SS, contribuindo para agilizar o processo e minimizar a ocorrência de erros. No que se refere ao mundo acadêmico, a ferramenta oferece recursos potenciais de apoio aos estudos fundamentados em dados dos currículos Lattes.

Cabe ainda destacar a contribuição teórica e prática à área de gerenciamento de projetos. Os detalhes dos procedimentos, os problemas, os erros e acertos, contemplam as especificidades e a complexidade da organização pesquisada. Esse conhecimento pode ser reutilizado como lições aprendidas, em projetos da Instituição e em outras de mesmo perfil.

A pouca disponibilidade de tempo e recursos determinou a redução da abrangência do ambiente pesquisado, sendo considerados apenas um Programa, uma área de conhecimento e um Estado da Federação. Os mesmos fatores restringiram o desenvolvimento das funcionalidades em modelos e protótipos, em vez de artefatos integrais. A abrangência restrita de dados e funcionalidades também limitou a aplicação da abordagem de gerenciamento do projeto. Alguns artefatos e técnicas previstos pelo modelo não foram contemplados. Outra limitação deste trabalho se refere ao fato de que os Cadernos de indicadores disponibilizados pela CAPES não são constantemente atualizados. Para este trabalho, foram utilizadas as versões mais recentes destes Cadernos, todavia contendo os dados referentes ao quadriênio anterior, finalizado no ano de 2012. Espera-se que tais documentos sejam atualizados e disponibilizados com maior frequência pela CAPES, para que possam ser geradas pela ferramenta ListaSucupira listas fidedignas contendo os dados dos professores que efetivamente fazem parte do Programa no momento das extrações, permitindo assim uma extração mais confiável de dados pelo ScriptSucupira, o que por sua vez permite o gerenciamento quase em tempo real dos cursos de Pós-Graduação por parte de seus gestores.

Por outro lado, os mesmos elementos que limitaram o trabalho indicam as possíveis oportunidades para continuá-lo. O caminho natural é a construção de um sistema de informação que implemente integralmente a ferramenta, em ambiente computacional robusto, usando um banco de dados de mercado e integrando um conjunto de dados mais abrangente. 


\section{Referências}

Akim, É. K., Mergulhão, R. C., \& Borrás, M. Á. A. (2013). Proposta de análise de falhas na coleta de informações para a avaliação de programas de pós-graduação baseada no FMEA. Revista Eletrônica Produção \& Engenharia, 5(1), 500-518.

Almeida, M. H. (2010, dezembro). A pós-graduação no Brasil: onde está e para onde poderia ir, em Plano Nacional de Pós-Graduação (PNPG) 2011-2020 Documentos Setoriais Volume II. DTI/CGD/CAPES.

Alves, A. D., Yanasse, H. H., \& Soma, N. Y. (2012). LattesMiner: uma linguagem de domínio específico para extração automática de informações da Plataforma Lattes. Apresentado em XII Workshop de computação aplicada, São José dos Campos.

Balbachevsky, E. (2005). A pós-graduação no Brasil: novos desafios para uma política bemsucedida. Os desafios da educação no Brasil. Rio de Janeiro: Nova Fronteira, 1, 285-314.

Bavaresco, J., Ferraz, R. R. N., Barro, F. C., \& Boeira, D. de A. R. (2015). Uso da ferramenta computacional Scriptsucupira como estratégia gerencial para acompanhamento da produção acadêmica em uma Instituição Educacional de Nível Superior. Revista de Gestão e Secretariado, 6(2), 1-18.

Bernheim, C. T., \& Chaui, M. de S. (2003). Challenges of the university in the knowledge society, five years after the World Conference on Higher Education. In Paper produced for the UNESCO Forum Regional Scientific Committee for Latin America and the Caribbean (UNESCO Forum Occasional Paper Series N 4).

Biancolino, C. A., Kniess, C. T., Maccari, E. A., \& Rabechini Jr., R. (2012). Protocolo para elaboração de relatos de produção técnica. Revista de Gestão e Projetos - GeP, 3(2), 294-307.

Cabral Neto, A. (2012). Mudanças contextuais e as novas regulações: repercussões no campo da política educacional. Revista Educação em Questão, 42(28), 7-40.

Campanario, M. de A., Maccari, E. A., da Silva, M. M., \& Santana, S. G. (2009). Desenvolvimento de um curso de mestrado profissional sob a perspectiva da gestão de projetos. Revista Brasileira de Gestão de Negócios, 11(33), 423-442.

CAPES/MEC. (2008). CAPES - História e missão. Recuperado de http://www.capes.gov.br /historia-e-missao

CAPES/MEC. (2010). Plano Nacional de Pós-Graduação (PNPG) 2011-2020 Volume I. DTI/CGD/CAPES.

CAPES/MEC. (2014a). CAPES - Sobre a Avaliação. Recuperado 21 de fevereiro de 2015, de http://www.capes.gov.br/avaliacao/sobre-a-avaliacao

CAPES/MEC. (2014b). CAPES - Entrada no SNPG: Propostas de cursos novos. Recuperado de http://www.capes.gov.br/avaliacao/entrada-no-snpg-propostas 
CAPES/MEC. (2014c). Coleta de Dados - Conceitos e orientações - Versão 1.5.

CAPES/MEC. (2014d). GEOCAPES - Sistema de Informações Georreferenciadas |CAPES. Recuperado de http://www.capes.gov.br/component /content/article/91-conteudoestatico/avaliacao-capes/6886-geocapes

Carvalho, M. M. de, \& Rabechini Jr., R. (2011). Fundamentos em Gestão de Projetos: Construindo Competências para Gerenciar Projetos ( $\left.3^{\circ} \mathrm{ed}\right)$. São Paulo: Atlas.

Christopoulos, T. P. (2004). Importância da estratégia na gestão de projetos de TI. In Anais do Congresso Anual de Tecnologia de Informação (Vol. 4, p. 1-15). São Paulo.

Cleland, D., \& Ireland, L. (2007). Project management: strategic design and implementation ( $5^{\circ}$ ed). McGraw Hill Professional.

Fernandez, D. J., \& Fernandez, J. D. (2008). Agile project management-Agilism versus traditional approaches. Journal of Computer Information Systems, 49(2), 10-17.

Ferraz, R. R. N., Quoniam, L. M., \& Maccari, E. A. (2014). A utilização da ferramenta Scriptlattes para extração e disponibilização on-line da produção acadêmica de um programa de stricto sensu em Administração. Revista Brasileira de Pós-Graduação, 11(24). http://doi.org/10.5748/9788599693100-11

Ferraz, R. R. N., Quoniam, L., \& Rezende, L. M. A. (2014). Avaliação de redes multidisciplinares com a ferramenta scriptlattes: os casos da nanotecnologia, da dengue e de um programa de pós-graduação stricto sensu em Administração. Encontros Bibli: revista eletrônica de biblioteconomia e ciência da informação, 19(40), 67-98.

Flick, U. (2009). An introduction to qualitative research. SAGE Publications.

Gerhardt, T. E., \& Silveira, D. T. (2009). Métodos de pesquisa (1ºd). Porto Alegre, RS: Plageder.

Gil, A. C. (2008). Como elaborar projetos de pesquisa (4º ed). São Paulo: Atlas.

Gil, A. C. (2010). Métodos e técnicas de pesquisa social (16º ed). São Paulo: Atlas.

Godoy, A. S. (1995). Introdução à pesquisa qualitativa e suas possibilidades. Revista de administração de empresas, 35(2), 57-63.

Guedes, C. A. (2001). Currículo Lattes perguntas e respostas. Recuperado de http://www.pucrs.campus2.br/manuais/dicas_lattes.pdf

Hanisch, B., \& Wald, A. (2012). A bibliometric view on the use of contingency theory in project management research. Project Management Journal, 43(3), 4-23. 
Junior, J. S. S., \& Quintella, R. H. (2005). Descoberta de conhecimento em bases de dados públicas: uma proposta de estruturação metodológica. Revista de Administração Pública, 39(5), 1077-1107.

Junior, L. J. M., \& Plonski, G. A. (2011). Gestão de projetos em empresas no Brasil: abordagem "tamanho único"? Gestão da Produção, 18(1), 1-12.

Kerzner, H. (2011). Gerenciamento de projetos - uma abordagem: sistêmica para planejamento, programação e controle $\left(10^{\mathrm{a}} \mathrm{ed}\right)$. São Paulo: Edgard Blucher.

Maccari. (2015). Gestão de pós-graduação por meio da ferramenta computacional scriptlattes (Tese de Livre Docência). USP, São Paulo.

Maccari, E. A., \& Teixeira, G. dos S. (2014). Estratégia e planejamento de projeto para acompanhamento de alunos egressos de programas de pós-graduação stricto sensu. Revista de Administração da UFSM, 7(1), 101-116.

Mainardes, E. W., Miranda, C. S., \& Correia, C. H. (2011). A gestão estratégica de instituições de ensino superior: um estudo multicaso. Contextus-Revista Contemporânea de Economia e Gestão, 9, p-19.

Marinho, M., Sampaio, S., Lima, T., \& de Moura, H. (2014). A guide to deal with uncertainties in software project management. International Journal of Computer Science \& Information Technology, 6(5), 1.

Maximiano, A. C. A. (2014). Administração de projetos: como transformar idéias em resultados ( $5^{\mathrm{a}}$ ed). São Paulo: Atlas.

MEC (2008). CAPES lança aplicativo gráfico para consulta única de dados. Recuperado de http://portal.mec.gov.br/index.php?id=14059\&option=com_content\&view =article

Mena-Chalco, J. P., \& Junior, R. C. M. (2009). ScriptLattes:an open-source knowledge extraction system from the Lattes platform. Journal of the Brazilian Computer Society, 15, 31 $-39$.

Mena-Chalco, J. P., \& Junior, R. M. C. (2011). Prospecção de dados acadêmicos de currículos Lattes através de ScriptLattes. Capítulo do livro Bibliometria e Cientometria: reflexões teóricas e interfaces (in press). São Carlos: Pedro \& João, 1-20.

Meyer Jr., V. (2008). Planejamento universitário: ato racional, político ou simbólico-um estudo de universidades brasileiras. Revista Alcance, 12(3), 373-390.

Moritz, G. de O., Pereira, M. F., Moritz, M. O., \& Maccari, E. A. (2013). A Pós-Graduação brasileira: evolução e principais desafios no ambiente de cenários prospectivos. Future Studies Research Journal: Trends and Strategies, 5(2), 03-34.

Neves, C., Martins, C. B., \& Leite, D. (2002). A estrutura e o funcionamento do ensino superior no Brasil. In A educação superior no Brasil (p. 24-37). Porto Alegre, RS: CAPES, UNESCO, UFRGS. 
Nigro, C. A., Ferraz, R. R. N., Quoniam, L., \& Alves, W. A. L. (2015). Uso da ferramenta computacional Scriptlattes-Scriptsucupira na prestação de contas anual e quadrienal da CAPES por um programa de pós-graduação stricto sensu em Direito. In Resultado - XVIII SemeAd. São Paulo. Recuperado de http://semead.com.br/resultado-xviii-semead/

Nigro, C. A., Ferraz, R. R. N., Quoniam, L., Alves, W. A. L., \& Messias, L. R. R. (2016). Prestação de contas anual e quadrienal à Capes por um programa de Pós-Graduação stricto sensu em Engenharia de Produção: utilização da ferramenta computacional ScriptlattesScriptsucupira. Revista PRISMA. COM, (29). Recuperado de http://revistas.ua.pt/index.php/prismacom/article/view/3641

Pacheco, R. C. dos S., \& Kern, V. M. (2001). Uma ontologia comum para a integração de bases de informações e conhecimento sobre ciência e tecnologia. Ci. Inf, 30(3), 56-63.

PMI. (2013). Um guia do conhecimento em gerenciamento de projectos (Guia PMBOK®) Quinta Edição (5 Edição). Newtown Square, PA: Project Management Institute, Inc.

Quoniam, L., \& Ferraz, R. R. N. (2014). Extração e disponibilização on-line de indicadores de desempenho e prospecção dos resultados das pesquisas em dengue realizadas pela comunidade científica brasileira por meio da utilização da ferramenta computacional Scriptlattes. Apresentado em XXXVIII Encontro da ANPAD.

Rolim, E. G., \& Amaral, L. H. (2007). A gestão da produção acadêmico-científica nas instituições de ensino superior: a experiência da Faculdade ce Ciências Médicas da Santa Casa de São Paulo. Arq Med Hosp Fac Cienc Med Santa Casa, (52(1)), 3-7.

Santos, C. (2003). Tradições e contradições da pós-graduação no Brasil. Educação e Sociedade, 24(83), 627-641.

Shenhar, A. J. (2012). What is Strategic Project Leadership? In PMI Global Congress Proceeding. Vancouver, Canada: PMI Canada.

Shenhar, A. J., \& Dvir, D. (2010). Reinventando gerenciamento de projetos. A Abordagem Diamante ao crescimento e inovação bem-sucedidos $\left(1^{\circ} \mathrm{ed}\right)$. M. Books.

Sidone, O. J. G. (2013). Análise espacial da produção e das redes de colaboração científica no Brasil: 1990-2010. Universidade de São Paulo.

Silva, H. A. S. da, Reina, D. R. M., Ensslin, S. R., \& Reina, D. (2012). Programas de pósgraduação em contabilidade: análise da produção científica e redes de colaboração. Revista de Contabilidade e Organizações, 6(14), 145-162.

Silva, R., Maccari, E., \& Quoniam, L. (2015). Uma ferramenta para apoiar a seleção de dados no processo de descoberta de conhecimento em bancos de dados de produção acadêmica. Revista Gestão \& Tecnologia, 15(1), 298-318. 
Soares, L. H. (2014). Gestão de instituições de ensino: o ensino superior privado e os novos parâmetros de perenidade. Universitas: Gestão e TI, 3(2).

Teixeira, G., \& Maccari, E. A. (2014). Desenvolvimento de uma sistemática para acompanhamento de alunos e egressos sob a perspectiva da gestão de projetos.

Theóphilo, C. R., \& Martins, G. A. (2009). Metodologia da investigação científica para ciências sociais aplicadas ( $2^{\circ}$ ed). São Paulo: Atlas.

Thiollent. (2009). Pesquisa-ação nas organizações (2º ed). São Paulo (SP): Atlas.

Thiollent. (2011). Metodologia da pesquisa-ação (18 ed). São Paulo: Cortez.

UNESCO. (1996, maio). UNESCO and an Information Society for All. UNESCO.

Williams, T. (2005). Assessing and moving on from the dominant project management discourse in the light of project overruns. Engineering Management, IEEE Transactions on, 52(4), 497-508.

Wysocki, R. K. (2013). Effective project management: traditional, agile, extreme ( $\left.7^{\circ} \mathrm{ed}\right)$. John Wiley \& Sons.

Yassuda, I. dos S. (2013). Artefatos de categorização de projetos espaciais e seleção de metodologias de gestão (Tese de Doutorado). INPE, São José dos Campos. 